Appl. Set-Valued Anal. Optim. 2 (2020), No. 1, pp. 35-48

Available online at http://asvao.biemdas.com

https://doi.org/10.23952/asvao.2.2020.1.03

\title{
ON PERIODIC OSCILLATIONS OF SOME POINTS OF A STRING WITH A NONLINEAR BOUNDARY CONDITION
}

\author{
MIKHAIL KAMENSKII*, NATALIA VOSKOVSKAYA, MARGARITA ZVEREVA \\ Faculty of Mathematics, Voronezh State University, Voronezh, Russia
}

\begin{abstract}
In this paper, we investigate an initial boundary value problem describing oscillation process with a hysteresis type boundary condition. The analogue of the d'Alembert formula is obtained. We study the possibility of periodic oscillations of some points of a string with such condition.
\end{abstract}

Keywords. Wave equation; String oscillations; Hysteresis; Periodic oscillations.

\section{INTRODUCTION}

There are many papers dedicated to the study of string oscillations with different types of conditions; see, e.g., $[4,6,7,8,9,10,13,14,15,18,19,20]$ and the references therein. In this paper, we investigate a boundary value problem describing oscillation process with a hysteresis type boundary condition. This kind of problems arises in a simulation of string oscillations, where the movement is restricted by a sleeve, which is concentrated at a boundary point. A case when time varying on the interval $0 \leq t \leq l$ was considered in $[13,20]$. In this paper, we consider a case that $t \in[0,+\infty)$. The analogue of the d'Alembert formula is obtained. The possibility of periodic oscillations of some points of a string with such condition is also investigated.

\section{PRELIMINARIES}

In this section, we recall some notions and definitions which will need in the sequel (details can be found in [17]).

Let $H$ be a Hilbert space. The inner product in $H$ is denoted by $\langle\cdot, \cdot\rangle$. For a closed convex set $C \subset H$ and $x \in C$, the set

$$
N_{C}(x)=\{\xi \in H:\langle\xi, c-x\rangle \leq 0 \quad \forall c \in C\}
$$

denotes the outward normal cone to $C$ at $x$.

Notice that we always have $0 \in N_{C}(x), N_{\{x\}}(x)=H$, and $N_{C}(x)=\{0\}$ for $x \in$ int $C$, where int $C$ is the interior of $C$. The last relation shows that the outward normal cone is non-trivial only for $x \in \partial C$, where $\partial C$ is the boundary of $C$.

\footnotetext{
${ }^{*}$ Corresponding author.

E-mail addresses: mikhailkamenski@mail.ru (M. Kamenskii), natashavskvskaja@rambler.ru (N. Voskovs kaya),margz@rambler.ru (M. Zvereva).

Received November 27, 2019; Accepted February 13, 2020.
}

(C)2020 Applied Set-Valued Analysis and Optimization 
Recall that the Hausdorff distance $d_{H}\left(C_{1}, C_{2}\right)$ between closed sets $C_{1}$ and $C_{2}$ is given by the formula

$$
d_{H}\left(C_{1}, C_{2}\right)=\max \left\{\sup _{x \in C_{2}} \operatorname{dist}\left(x, C_{1}\right), \sup _{x \in C_{1}} \operatorname{dist}\left(x, C_{2}\right)\right\}
$$

Consider so called "sweeping process"

$$
\begin{gathered}
-u^{\prime}(t) \in N_{C(t)}(u(t)) \text { for a.e. } t \in[0, T], \\
u(0)=u_{0} \in C(0) .
\end{gathered}
$$

A function $u:[0, T] \rightarrow H$ is called a solution of sweeping process (2.1)-(2.2) if

(a) $u(0)=u_{0}$;

(b) $u(t) \in C(t)$ for all $t \in[0, T]$;

(c) $u$ is differentiable at a.e. $t \in[0, T]$;

(d) $-u^{\prime}(t) \in N_{C(t)}(u(t))$ at a.e. $t \in[0, T]$.

There are many papers dedicated to sweeping processes; see, e.g., $[1,2,3,5,11,12,16,17]$ and the references therein. The following two theorems are useful for our main results.

Theorem 2.1. [17, Theorem 2] (Existence) Assume that the map $t \rightarrow C(t)$ satisfies

$$
d_{H}(C(t), C(s)) \leq L|t-s|
$$

and $C(t) \subset H$ is nonempty, closed and convex for every $t \in[0, T]$. Let $u_{0} \in C(0)$. Then there exists a solution $u:[0, T] \rightarrow H$ of (2.1),(2.2) which is Lipschitz continuous with constant $L$. In particular, $\left|u^{\prime}(t)\right| \leq L$ for almost every $t \in[0, T]$.

Theorem 2.2. [17, Theorem 3] (Uniqueness) The solution of (2.1),(2.2) is unique in the class of absolutely continuous functions.

\section{A PROBLEM WITH A HYSTERESIS TYPE BOUNDARY CONDITION}

Suppose that a string is located along the segment $[0, l]$. Let $u(x, t)$ be a deviation from the equilibrium position at the time $t$. Assume that the left end of the string is rigidly fixed, i.e., $u(0, t)=0$. The right end of the string moves along a vertical needle (without friction) inside a sleeve, represented by $[-h, h]$, where $h>0$. While $|u(l, t)|<h$, the right end of the string inside of the sleeve remains free, i.e., $u_{x}^{\prime}(l, t)=0$. If the string reaches the boundary points of the sleeve, then the conditions $u(l, t)=h$, or $u(l, t)=-h$, respectively, are satisfied. Notice that we consider the case where the sleeve can move in perpendicular to the axis $O x$ direction and its movement is given by

$$
C(t)=[-h, h]+\xi(t)
$$

Suppose that the string velocity is zero at the initial time $t=0$ and the string form is determined by the function $\varphi(\cdot) \in W_{2}^{1}[0, l]$, where $\varphi(0)=0, \varphi(l) \in C(0)$. 
The mathematical model of such problem can be described as

$$
\left\{\begin{array}{l}
\frac{\partial^{2} u}{\partial x^{2}}=\frac{\partial^{2} u}{\partial t^{2}} \\
u(x, 0)=\varphi(x) \\
\frac{\partial u}{\partial t}(x, 0)=0 \\
u(0, t)=0 \\
u(l, t) \in C(t) \\
-u_{x}^{\prime}(l, t) \in N_{C(t)}(u(l, t))
\end{array}\right.
$$

where the set $N_{C(t)}(u(l, t))$ is the outward normal cone to $C(t)$ at $u(l, t)$, which is defined as

$$
N_{C(t)}(u(l, t))=\left\{\xi \in R^{1}: \xi \cdot(c-u(l, t)) \leq 0 \quad \forall c \in C(t)\right\} .
$$

Notice if $u(l, t)$ is an interior point of $C(t)$, then $N_{C(t)}(u(l, t))=\{0\}$; if $u(l, t)=-h+\xi(t)$, then

$$
N_{C(t)}(u(l, t))=(-\infty, 0]
$$

if $u(l, t)=h+\xi(t)$, then

$$
N_{C(t)}(u(l, t))=[0,+\infty) .
$$

The condition $-u_{x}^{\prime}(l, t) \in N_{C(t)}(u(l, t))$ means that if $u(l, t)$ is an interior point of $C(t)$, then $u_{x}^{\prime}(l, t)=0$, i.e., the oscillation process is such as for a string with a free right end (see [18]); when the right end of the string contacts with the boundary sleeve point, the right end of the string is not free any more: there is the force $f(t)$, which blocks this end so $-u_{x}^{\prime}(l, t)=-f(t) \in$ $N_{C(t)}(u(l, t))$. Consider a special class of functions, introduced for the first time by Il'in in $[8,9]$. Let $Q_{T}$ be the rectangle $Q_{T}=[0 \leq x \leq l] \times[0 \leq t \leq T]$. As in [8,9], we suppose that $u$ belongs to the class $\widehat{W}_{2}^{1}\left(Q_{T}\right)$ if $u(x, t)$ is continuous in the closed rectangle $Q_{T}$ and has in this rectangle both generalized partial derivatives $u_{x}^{\prime}(x, t)$ and $u_{t}^{\prime}(x, t)$, which belong to the class $L_{2}\left(Q_{T}\right)$. Moreover, $u_{x}^{\prime}(\cdot, t)$ belongs to the class $L_{2}[0 \leq x \leq l]$ for every fixed $t$ of the segment $[0, T]$ and $u_{t}^{\prime}(x, \cdot)$ belongs to the class $L_{2}[0 \leq t \leq T]$, for any fixed $x$ of the segment $[0, l]$.

By a solution of (3.2) we that a function $u(x, t)$ such that conditions $u(l, t) \in C(t)$ and $u(0, t)=$ 0 hold for all $t$; conditions $-u_{x}^{\prime}(l, t) \in N_{C(t)}(u(l, t))$ and $\frac{\partial u}{\partial t}(x, 0)=0$ hold for almost every $t$; the condition $u(x, 0)=\varphi(x)$ holds for all $x \in[0, l]$; for all $T>0 u \in \widehat{W}_{2}^{1}\left(Q_{T}\right)$ and the integral identity

$$
\begin{aligned}
& \int_{0}^{l} \int_{0}^{T} u(x, t)\left[\Psi_{t t}(x, t)-\Psi_{x x}(x, t)\right] d x d t+\int_{0}^{l} \Psi_{t}^{\prime}(x, 0) \varphi(x) d x-\int_{0}^{T} \Psi(l, t) u_{x}^{\prime}(l, t) d t \\
& \quad+\int_{0}^{T} \Psi_{x}^{\prime}(l, t) u(l, t) d t=0
\end{aligned}
$$

holds for any function $\Psi \in C^{2}\left(Q_{T}\right)$, which satisfies the conditions $\Psi(0, t)=0, \Psi(x, T)=0$, $\Psi_{t}^{\prime}(x, T)=0$.

Theorem 3.1. Assume that the function $\xi(t)$ satisfies the Lipschitz condition for all $t \geq 0$, and the function $\varphi(x)$ satisfies the Lipschitz condition for all $x \in[0, l]$. Then a solution of problem (3.2) can be represented as

$$
u(x, t)=\frac{\Phi(x-t)+\Phi(x+t)}{2}
$$


where the function $\Phi(x)$ has the following form:

if $x \in[0, l]$, then $\Phi(x)=\varphi(x)$;

if $x \in[(i+1) l,(i+2) l]$ and $i$ is an even number, then

$$
\Phi(x)=2 \cdot \sum_{k=0}^{\frac{i}{2}} g_{2 k}(x-(i+1-2 k) l)-\varphi((i+2) l-x)
$$

if $x \in[(i+1) l,(i+2) l]$ and $i$ is an odd number, then

$$
\begin{gathered}
\Phi(x)=2 \cdot \sum_{k=1}^{\frac{i+1}{2}} g_{2 k-1}(x-(i+2-2 k) l)+\varphi(x-(i+1) l) ; \\
\Phi(-x)=-\Phi(x),
\end{gathered}
$$

where functions $g_{0}(t)$ and $g_{1}(t)$ are solutions of the problems

$$
\left\{\begin{array}{l}
-g_{0}^{\prime}(t) \in N_{C(t)}\left(g_{0}(t)\right)+\varphi^{\prime}(l-t), t \in[0, l], \\
g_{0}(0)=\varphi(l)
\end{array}\right.
$$

and

$$
\left\{\begin{array}{l}
-g_{1}^{\prime}(t) \in N_{C(t)}\left(g_{1}(t)\right)+\varphi^{\prime}(t-l), t \in[l, 2 l], \\
g_{1}(l)=g_{0}(l) .
\end{array}\right.
$$

Functions $g_{i}(t)$ for even numbers $i \geq 2$ are solutions of the problems

$$
\left\{\begin{array}{l}
-g_{i}^{\prime}(t) \in N_{C(t)}\left(g_{i}(t)\right)+2 \sum_{k=0}^{\frac{i-2}{2}} g_{2 k}^{\prime}(t-i l+2 k l)+\varphi^{\prime}(i l+l-t), t \in[i l,(i+1) l], \\
g_{i}(i l)=g_{i-1}(i l),
\end{array}\right.
$$

and for odd numbers $i \geq 3$ are solutions of the problems

$$
\left\{\begin{array}{l}
-g_{i}^{\prime}(t) \in N_{C(t)}\left(g_{i}(t)\right)+2 \sum_{k=1}^{\frac{i-1}{2}} g_{2 k-1}^{\prime}(t-l-i l+2 k l)+\varphi^{\prime}(t-i l), t \in[i l,(i+1) l], \\
g_{i}(i l)=g_{i-1}(i l) .
\end{array}\right.
$$

Proof. Suppose formally that a solution of problem (3.2) has form (3.4). Then $u(x, 0)=\Phi(x)=$ $\varphi(x)$, where $x \in[0, l]$. Since $\varphi(x)$ satisfies the Lipschitz condition, we get $\Phi \in W_{2}^{1}[0, l]$. Since $u(x, 0)=0$ we have $\Phi(x)=-\Phi(-x)$. Let us consider the condition $-u_{x}^{\prime}(l, t) \in N_{C(t)}(u(l, t))$. If the solution of problem (3.2) has form (3.4), then

$$
-u_{t}^{\prime}(l, t) \in N_{C(t)}(u(l, t))+\Phi^{\prime}(l-t) .
$$

Consider the case when $t \in[0, l]$. We denote by $g_{0}(t)=u(l, t)$. Then $g_{0}(t)$ is a solution of the problem

$$
\left\{\begin{array}{l}
-\frac{d}{d t} g_{0}(t) \in N_{C(t)}\left(g_{0}(t)\right)+\varphi^{\prime}(l-t) \\
g_{0}(0)=\varphi(l), t \in[0, l]
\end{array}\right.
$$

Let us show that problem (3.6) has a unique solution. Consider the function

$$
w(t)=g_{0}(t)+\int_{0}^{t} \varphi^{\prime}(l-s) d s
$$


and the set

$$
D(t)=C(t)+\int_{0}^{t} \varphi^{\prime}(l-s) d s .
$$

Notice that $N_{C(t)}\left(g_{0}(t)\right)=N_{D(t)}(w(t))$. Thus we have the problem

$$
-\frac{d}{d t} w(t) \in N_{D(t)}(w(t)), w(0)=\varphi(l) \in D(0), t \in[0, l] .
$$

According to Theorems 2.1 and 2.2, this problem has a unique solution $w(t)$, which is defined on the whole interval $[0, l]$. The function $w(t)$ is absolutely continuous and its derivative is bounded almost everywhere. Thus problem (3.6) has a unique solution $g_{0}(t)$, where $g_{0}(t) \in C(t)$ and $g_{0}(t)$ satisfies the Lipschitz condition. Therefore

$$
\Phi(l-t)+\Phi(l+t)=2 g_{0}(t)
$$

and then

$$
\Phi(x)=2 g_{0}(x-l)-\varphi(2 l-x),
$$

where $x \in[l, 2 l]$. Notice that $\Phi(x)$ satisfies the Lipschitz condition on $[l, 2 l]$. So, $\Phi \in W_{2}^{1}[l, 2 l]$. Let us show that $\Phi(l-0)=\Phi(l+0)$. We have $\Phi(l-0)=\varphi(l)$ and

$$
\Phi(l+0)=2 g_{0}(0)-\varphi(l)=2 \varphi(l)-\varphi(l)=\varphi(l) .
$$

Consider a case when $t \in[l, 2 l]$. Define $g_{1}(t)=u(l, t)$. Consider a problem

$$
\left\{\begin{array}{l}
-\frac{d}{d t} g_{1}(t) \in N_{C(t)}\left(g_{1}(t)\right)+\Phi^{\prime}(l-t), \\
g_{1}(l)=g_{0}(l), t \in[l, 2 l]
\end{array}\right.
$$

Notice that $\Phi(l-t)=-\varphi(t-l)$. Thus we have a problem

$$
\left\{\begin{array}{l}
-\frac{d}{d t} g_{1}(t) \in N_{C(t)}\left(g_{1}(t)\right)+\varphi^{\prime}(t-l), \\
g_{1}(l)=g_{0}(l), t \in[l, 2 l] .
\end{array}\right.
$$

Similarly to problem (3.6) we obtain that the last problem has a unique solution $g_{1}(t)$, where $g_{1}(t) \in C(t)$ and $g_{1}(t)$ satisfies the Lipschitz condition. Thus we define $\Phi(x)$, where $x \in[2 l, 3 l]$ as

$$
\Phi(x)=2 g_{1}(x-l)+\varphi(x-2 l) .
$$

Notice that $\Phi(x)$ satisfies the Lipschitz condition on $[2 l, 3 l]$. Hence, $\Phi \in W_{2}^{1}[2 l, 3 l]$. Let us show that $\Phi(2 l-0)=\Phi(2 l+0)$. We have $\Phi(2 l-0)=2 g_{0}(l)-\varphi(0)=2 g_{0}(l)$ and $\Phi(2 l+0)=$ $2 g_{1}(l)+\varphi(0)=2 g_{0}(l)$.

Similarly, we consider the case when $t \in[2 l, 3 l]$. Define $g_{2}(t)=u(l, t)$. Then $g_{2}(t)$ is a solution of the problem

$$
\left\{\begin{array}{l}
-\frac{d}{d t} g_{2}(t) \in N_{C(t)}\left(g_{2}(t)\right)+2 g_{0}^{\prime}(t-2 l)+\varphi^{\prime}(3 l-t), \\
g_{2}(2 l)=g_{1}(2 l), t \in[2 l, 3 l] .
\end{array}\right.
$$

Hence,

$$
\Phi(x)=2 g_{2}(x-l)+2 g_{0}(x-3 l)-\varphi(4 l-x),
$$

where $x \in[3 l, 4 l]$. 
Consider the case when $t \in[3 l, 4 l]$. Define $g_{3}(t)=u(l, t)$. Then $g_{3}(t)$ is a solution of the problem

$$
\left\{\begin{array}{l}
-\frac{d}{d t} g_{3}(t) \in N_{C(t)}\left(g_{3}(t)\right)+2 g_{1}^{\prime}(t-2 l)+\varphi^{\prime}(t-3 l), \\
g_{3}(3 l)=g_{2}(3 l), t \in[3 l, 4 l]
\end{array}\right.
$$

and

$$
\Phi(x)=2 g_{3}(x-l)+2 g_{1}(x-3 l)+\varphi(x-4 l),
$$

where $x \in[4 l, 5 l]$. Let us show that if $x \in[(i+1) l,(i+2) l]$ and $i$ is an even number, then

$$
\Phi(x)=2 \cdot \sum_{k=0}^{\frac{i}{2}} g_{2 k}(x-(i+1-2 k) l)-\varphi((i+2) l-x)
$$

if $x \in[(i+1) l,(i+2) l]$ and $i$ is an odd number, then

$$
\Phi(x)=2 \cdot \sum_{k=1}^{\frac{i+1}{2}} g_{2 k-1}(x-(i+2-2 k) l)+\varphi(x-(i+1) l),
$$

where functions $g_{i}(t)$ for even numbers $i \geq 2$ are solutions of the problems

$$
\left\{\begin{array}{l}
-g_{i}^{\prime}(t) \in N_{C(t)}\left(g_{i}(t)\right)+2 \sum_{k=0}^{\frac{i-2}{2}} g_{2 k}^{\prime}(t-i l+2 k l)+\varphi^{\prime}(i l+l-t), t \in[i l,(i+1) l], \\
g_{i}(i l)=g_{i-1}(i l),
\end{array}\right.
$$

and for odd numbers $i \geq 3$ are solutions of the problems

$$
\left\{\begin{array}{l}
-g_{i}^{\prime}(t) \in N_{C(t)}\left(g_{i}(t)\right)+2 \sum_{k=1}^{\frac{i-1}{2}} g_{2 k-1}^{\prime}(t-l-i l+2 k l)+\varphi^{\prime}(t-i l), t \in[i l,(i+1) l], \\
g_{i}(i l)=g_{i-1}(i l) .
\end{array}\right.
$$

For $i=2,3$ the assertion is proved. Let it hold for $i \leq m$ and let us show it for $i=m+1$. Consider the case when $m$ is an even number. Let us show that

$$
\Phi(x)=2 \sum_{k=1}^{\frac{m+2}{2}} g_{2 k-1}(x-(m+3-2 k) l)+\varphi(x-(m+2) l),
$$

where $x \in[(m+2) l,(m+3) l]$. Define $u(l, t)=g_{m+1}(t)$, where $t \in[(m+1) l,(m+2) l]$. Then

$$
-g_{m+1}^{\prime}(t) \in N_{C(t)}\left(g_{m+1}(t)\right)+\Phi^{\prime}(l-t) .
$$

Since

$$
\Phi^{\prime}(l-t)=2 \cdot \sum_{k=1}^{\frac{m}{2}} g_{2 k-1}^{\prime}(t-l-(m+1-2 k) l)+\varphi^{\prime}(t-l-m l)
$$

we have

$$
-g_{m+1}^{\prime}(t) \in N_{C(t)}\left(g_{m+1}(t)\right)+2 \cdot \sum_{k=1}^{\frac{m}{2}} g_{2 k-1}^{\prime}(t-2 l-m+2 k l)+\varphi^{\prime}(t-l-m l) .
$$

Notice that

$$
g_{m+1}((m+1) l)=\frac{\Phi((2+m) l)-\Phi(m l)}{2} .
$$


Since $\Phi((m+2) l)=2 \sum_{k=0}^{\frac{m}{2}} g_{2 k}(l+2 k l)$ and $\Phi(m l)=2 \sum_{k=0}^{\frac{m-2}{2}} g_{2 k}(l+2 k l)$, we have $g_{m+1}((m+$ $1) l)=g_{m}((m+1) l)$. The problem

$$
\left\{\begin{array}{l}
-g_{m+1}^{\prime}(t) \in N_{C(t)}\left(g_{m+1}(t)\right)+2 \cdot \sum_{k=1}^{\frac{m}{2}} g_{2 k-1}^{\prime}(t-2 l-m+2 k l)+\varphi^{\prime}(t-l-m l), \\
g_{m+1}((m+1) l)=g_{m}((m+1) l)
\end{array}\right.
$$

has a unique solution $g_{m+1}(t)$, which is defined on the whole interval $[(m+1) l,(m+2) l]$. Then

$$
g_{m+1}(t)=\frac{\Phi(l-t)+\Phi(l+t)}{2}
$$

and we have

$$
\Phi(x)=2 g_{m+1}(x-l)-\Phi(2 l-x),
$$

where $x \in[(m+2) l,(m+3) l]$. Since $\Phi(2 l-x)=-\Phi(x-2 l)$ and

$$
\Phi(x-2 l)=2 \sum_{k=1}^{\frac{m}{2}} g_{2 k-1}(x-3 l-m l+2 k l)+\varphi(x-2 l-m l),
$$

we have

$$
\begin{aligned}
\Phi(x) & =2 g_{m+1}(x-l)+2 \sum_{k=1}^{\frac{m}{2}} g_{2 k-1}(x-3 l-m l+2 k l)+\varphi(x-2 l-m l) \\
& =2 \sum_{k=1}^{\frac{m+2}{2}} g_{2 k-1}(x-3 l-m l+2 k l)+\varphi(x-2 l-m l) .
\end{aligned}
$$

Other cases can be analyzed in the same way. Thus we define $\Phi(x)$ for all $x \in R$. Let us show that the function $u(x, t)$, which is defined by equality (3.4), is the solution of problem (3.2), where the function $\Phi(x)$ is defined above. Notice that the function $u \in \widehat{W}_{2}^{1}\left(Q_{T}\right)$ for all $T>0$, because $\Phi(x)$ is continuous on $x \in R$ and $\Phi \in W_{2}^{1}[i l,(i+1) l]$ for all $i=0,1,2, \ldots$ Since $u(l, t)=g(t)$, where $g(t)=g_{i}(t), t \in[i l,(i+1) l], g_{i}(i l)=g_{i-1}(i l)$ and $g_{i}(t) \in C(t)$, we conclude that $u(l, t) \in C(t)$ for all $t \geq 0$. Notice that the condition $u(0, t)=0$ holds for all $t ; \frac{\partial u}{\partial t}(x, 0)=0$ holds for almost every $t ; u(x, 0)=\varphi(x)$ holds for all $x \in[0, l]$. Notice that, for all $t \geq 0$,

$$
\Phi(l-t)+\Phi(l+t)=2 g(t) .
$$

Thus, for almost every $t$,

$$
-\Phi^{\prime}(l-t)+\Phi^{\prime}(l+t)=2 g^{\prime}(t) .
$$

Since $-g^{\prime}(t) \in N_{C(t)}(g(t))+\Phi^{\prime}(l-t)$, we have

$$
-u_{x}^{\prime}(l, t)=\frac{-\Phi^{\prime}(l-t)-\Phi^{\prime}(l+t)}{2}=-g^{\prime}(t)-\Phi^{\prime}(l-t) \in N_{C(t)}(g(t))=N_{C(t)}(u(l, t)) .
$$


Let us show that integral equality (3.3) holds for any function $\Psi \in C^{2}\left(Q_{T}\right)$, which satisfies the conditions $\Psi(0, t)=0, \Psi(x, T)=0, \Psi_{t}^{\prime}(x, T)=0$. We have

$$
\begin{aligned}
& \int_{0}^{l}\left(\int_{0}^{T} u(x, t) \Psi_{t t}(x, t) d t\right) d x-\int_{0}^{T}\left(\int_{0}^{l} u(x, t) \Psi_{x x}(x, t) d x\right) d t \\
& \quad+\int_{0}^{l} \Psi_{t}^{\prime}(x, 0) \varphi(x) d x-\int_{0}^{T} \Psi(l, t) u_{x}^{\prime}(l, t) d t+\int_{0}^{T} \Psi_{x}^{\prime}(l, t) u(l, t) d t \\
& =\int_{0}^{l}\left(u(x, T) \Psi_{t}^{\prime}(x, T)-u(x, 0) \Psi_{t}^{\prime}(x, 0)\right) d x-\int_{0}^{l} \int_{0}^{T} u_{t}^{\prime}(x, t) \Psi_{t}^{\prime}(x, t) d t d x \\
& \quad-\int_{0}^{T}\left(\Psi_{x}^{\prime}(l, t) u(l, t)-\Psi_{x}^{\prime}(0, t) u(0, t)\right) d t \\
& \quad+\int_{0}^{T} \int_{0}^{l} u_{x}^{\prime}(x, t) \Psi_{x}^{\prime}(x, t) d x d t+\int_{0}^{l} \Psi_{t}^{\prime}(x, 0) \varphi(x) d x \\
& \quad-\int_{0}^{T} \Psi(l, t) u_{x}^{\prime}(l, t) d t+\int_{0}^{T} \Psi_{x}^{\prime}(l, t) u(l, t) d t .
\end{aligned}
$$

We need to prove that

$$
\int_{0}^{T} \int_{0}^{l} u_{x}^{\prime}(x, t) \Psi_{x}^{\prime}(x, t) d x d t-\int_{0}^{T} \int_{0}^{l} u_{t}^{\prime}(x, t) \Psi_{t}^{\prime}(x, t) d x d t=\int_{0}^{T} \Psi(l, t) u_{x}^{\prime}(l, t) d t .
$$

According to (3.4), we have

$$
\begin{aligned}
\frac{1}{2} \int_{0}^{T} \int_{0}^{l}\left(\Phi^{\prime}(x-t)-\Phi^{\prime}(x+t)\right) \Psi_{x}^{\prime}(x, t) d x d t-\frac{1}{2} \int_{0}^{l} \int_{0}^{T}\left(\Phi^{\prime}(x+t)-\Phi^{\prime}(x-t)\right) \Psi_{t}^{\prime}(x, t) d t d x \\
=\frac{1}{2} \int_{0}^{l}\left(\Psi_{x}^{\prime}(x, T)(\Phi(x+T)-\Phi(x-T))-\Psi_{x}^{\prime}(x, 0)(\Phi(x)-\Phi(x))\right) d x \\
\quad-\frac{1}{2} \int_{0}^{l} \int_{0}^{T}(\Phi(x+t)-\Phi(x-t)) \Psi_{x t}(x, t) d t d x \\
\quad-\frac{1}{2} \int_{0}^{T}\left(\Psi_{t}^{\prime}(l, t)(\Phi(l+t)-\Phi(l-t))-\Psi_{t}^{\prime}(0, t)(\Phi(t)-\Phi(-t))\right) d t \\
\quad+\frac{1}{2} \int_{0}^{l} \int_{0}^{T}(\Phi(x+t)-\Phi(x-t)) \Psi_{x t}(x, t) d t d x \\
=-\frac{1}{2} \int_{0}^{T} \Psi_{t}^{\prime}(l, t)(\Phi(l+t)-\Phi(l-t)) d t .
\end{aligned}
$$

On the other hand, one has

$$
\begin{aligned}
\int_{0}^{T} \Psi(l, t) u_{x}^{\prime}(l, t) d t= & \frac{1}{2} \int_{0}^{T} \Psi(l, t)\left(\Phi^{\prime}(l-t)+\Phi^{\prime}(l+t)\right) d t \\
= & \frac{1}{2}(\Psi(l, T)(\Phi(l+T)-\Phi(l-T))-\Psi(l, 0)(\Phi(l)-\Phi(l))) \\
& -\frac{1}{2} \int_{0}^{T}(\Phi(l+t)-\Phi(l-t)) \Psi_{t}^{\prime}(l, t) d t \\
= & -\frac{1}{2} \int_{0}^{T}(\Phi(l+t)-\Phi(l-t)) \Psi_{t}^{\prime}(l, t) d t .
\end{aligned}
$$

The theorem is proved. 
Remark 3.1. Notice that problem (3.2) has a unique solution. Assume that $\varphi(l) \in(-h+$ $\xi(0), h+\xi(0))$. Then the oscillation process occurs as for the string with a free end for all $t \in\left[0, t_{1}\right]$, and the string form is the solution of the problem

$$
\left\{\begin{array}{l}
\frac{\partial^{2} u}{\partial x^{2}}=\frac{\partial^{2} u}{\partial t^{2}}, \quad 0<x<l, 0<t<t_{1} \\
u(x, 0)=\varphi(x) \\
\frac{\partial u}{\partial t}(x, 0)=0 \\
u(0, t)=0 \\
u_{x}^{\prime}(l, t)=0
\end{array}\right.
$$

According to [10], the last problem has a unique solution $u(x, t)$. The relation $u\left(l, t_{1}\right)= \pm h+$ $\xi(t)$ holds at the moment $t_{1}$, and for all $t \in\left[t_{1}, t_{2}\right]$ a string form is a solution of a problem

$$
\left\{\begin{array}{l}
\frac{\partial^{2} v}{\partial x^{2}}=\frac{\partial^{2} v}{\partial t^{2}}, \quad 0<x<l, t_{1}<t<t_{2} \\
v\left(x, t_{1}\right)=u\left(x, t_{1}\right) \\
\frac{\partial v}{\partial t}\left(x, t_{1}\right)=u_{t}^{\prime}\left(x, t_{1}\right) \\
v(0, t)=0 \\
v(l, t)=-h+\xi(t)
\end{array}\right.
$$

or

$$
\left\{\begin{array}{l}
\frac{\partial^{2} v}{\partial x^{2}}=\frac{\partial^{2} v}{\partial t^{2}}, \quad 0<x<l, t_{1}<t<t_{2} \\
v\left(x, t_{1}\right)=u\left(x, t_{1}\right) \\
\frac{\partial v}{\partial t}\left(x, t_{1}\right)=u_{t}^{\prime}\left(x, t_{1}\right) \\
v(0, t)=0 \\
v(l, t)=h+\xi(t)
\end{array}\right.
$$

Each of the above problems has a unique solution for every $t \in\left[t_{1}, t_{2}\right]$. By a similar reasoning, we get that the original problem has a unique solution.

\section{PERIODiC OSCILlations OF SOME POINTS OF A STRING}

Let us study the possibility of periodic oscillations of some points of a string which is described by the problem

$$
\left\{\begin{array}{l}
\frac{\partial^{2} u}{\partial x^{2}}=\frac{\partial^{2} u}{\partial t^{2}}, \\
u(x, 0)=0 \\
\frac{\partial u}{\partial t}(x, 0)=0, \\
u(0, t)=0, \\
u(l, t) \in C(t), \\
-u_{x}^{\prime}(l, t) \in N_{C(t)}(u(l, t)),
\end{array}\right.
$$


where $C(t)=[-h, h]+\xi(t)$ and $\xi(t)$ is a $l$-periodic function defined by the formula

$$
\xi(t)= \begin{cases}\frac{8 h}{l} t, & t \in\left[0, \frac{l}{4}\right], \\ \frac{-8 h}{l}\left(t-\frac{l}{2}\right), & t \in\left[\frac{l}{4}, \frac{3 l}{4}\right], \\ \frac{8 h}{l}(t-l), & t \in\left[\frac{3 l}{4}, l\right] .\end{cases}
$$

Notice that $\xi(t)$ satisfies the Lipschitz condition with constant $L=\frac{8 h}{l}$. According to Theorem 3.1 , the last problem has a unique solution $u(x, t)$, where

$$
u(x, t)=\frac{\Phi(x-t)+\Phi(x+t)}{2} .
$$

The function $\Phi(x)$ has the following form:

if $x \in[0, l]$, then $\Phi(x)=0$;

if $x \in[(i+1) l,(i+2) l]$ and $i$ is an even number then

$$
\Phi(x)=2 \cdot \sum_{k=0}^{\frac{i}{2}} g_{2 k}(x-(i+1-2 k) l)
$$

if $x \in[(i+1) l,(i+2) l]$ and $i$ is an odd number, then

$$
\begin{gathered}
\Phi(x)=2 \cdot \sum_{k=1}^{\frac{i+1}{2}} g_{2 k-1}(x-(i+2-2 k) l) ; \\
\Phi(-x)=-\Phi(x)
\end{gathered}
$$

where functions $g_{0}(t)$ and $g_{1}(t)$ are solutions of the problems

$$
\left\{\begin{array}{l}
-g_{0}^{\prime}(t) \in N_{C(t)}\left(g_{0}(t)\right), t \in[0, l], \\
g_{0}(0)=\varphi(l)
\end{array}\right.
$$

and

$$
\left\{\begin{array}{l}
-g_{1}^{\prime}(t) \in N_{C(t)}\left(g_{1}(t)\right), t \in[l, 2 l], \\
g_{1}(l)=g_{0}(l) .
\end{array}\right.
$$

Functions $g_{i}(t)$ for even numbers $i \geq 2$ are solutions of the problems

$$
\left\{\begin{array}{l}
-g_{i}^{\prime}(t) \in N_{C(t)}\left(g_{i}(t)\right)+2 \sum_{k=0}^{\frac{i-2}{2}} g_{2 k}^{\prime}(t-i l+2 k l), t \in[i l,(i+1) l], \\
g_{i}(i l)=g_{i-1}(i l),
\end{array}\right.
$$

and for odd number $i \geq 3$ are solutions of the problems

$$
\left\{\begin{array}{l}
-g_{i}^{\prime}(t) \in N_{C(t)}\left(g_{i}(t)\right)+2 \sum_{k=1}^{\frac{i-1}{2}} g_{2 k-1}^{\prime}(t-l-i l+2 k l), t \in[i l,(i+1) l], \\
g_{i}(i l)=g_{i-1}(i l) .
\end{array}\right.
$$


Consider problem (4.2). Notice that a solution of the last problem can be represented as

$$
g_{0}(t)= \begin{cases}0, & t \in\left[0, \frac{l}{8}\right], \\ \xi(t)-h, & t \in\left[\frac{l}{8}, \frac{l}{4}\right], \\ h, & t \in\left[\frac{l}{4}, \frac{l}{2}\right] \\ \xi(t)+h, & t \in\left[\frac{l}{2}, \frac{3 l}{4}\right], \\ -h, & t \in\left[\frac{3 l}{4}, l\right] .\end{cases}
$$

Consider problem (4.3). Notice that a solution of the last problem can be represented as

$$
g_{1}(t)= \begin{cases}\xi(t)-h, & t \in\left[l, \frac{5 l}{4}\right] \\ h, & t \in\left[\frac{5 l}{4}, \frac{3 l}{2}\right] \\ \xi(t)+h, & t \in\left[\frac{3 l}{2}, \frac{7 l}{4}\right] \\ -h, & t \in\left[\frac{7 l}{4}, 2 l\right] .\end{cases}
$$

Let us show that, for all $i \in N$,

$$
g_{i}(t)= \begin{cases}\xi(t)-h, & t \in\left[i l, \frac{l(4 i+1)}{4}\right], \\ h, & t \in\left[\frac{l(4 i+1)}{4}, \frac{l(2 i+1)}{2}\right], \\ \xi(t)+h, & t \in\left[\frac{l(2 i+1)}{2}, \frac{l(4 i+3)}{4}\right], \\ -h, & t \in\left[\frac{l(4 i+3)}{4}, l(i+1)\right] .\end{cases}
$$

For $i=1$, the assertion is proved. Let it hold for $i \leq n$. Let us show it for $i=n+1$. Consider the case when $n$ is an even number, i.e., $\mathrm{n}=2 \mathrm{~m}$. Let us show that

$$
g_{2 m+1}(t)= \begin{cases}\xi(t)-h, & t \in\left[2 m l+l, 2 m l+\frac{5 l}{4}\right], \\ h, & t \in\left[2 m l+\frac{5 l}{4}, 2 m l+\frac{3 l}{2}\right], \\ \xi(t)+h, & t \in\left[2 m l+\frac{3 l}{2}, 2 m l+\frac{7 l}{4}\right], \\ -h, & t \in\left[2 m l+\frac{7 l}{4}, 2 m l+2 l\right] .\end{cases}
$$

We have

Setting

$$
\left\{\begin{array}{l}
-g_{2 m+1}^{\prime}(t) \in N_{C(t)}\left(g_{2 m+1}(t)\right)+2 \sum_{k=1}^{m} g_{2 k-1}^{\prime}(t-2 l-2 m l+2 k l), \\
g_{2 m+1}((2 m+1) l)=-h, \quad t \in[(2 m+1) l,(2 m+2) l] .
\end{array}\right.
$$

$$
v(t)=g_{2 m+1}(t)+2 \int_{(2 m+1) l}^{t} \sum_{k=1}^{m} g_{2 k-1}^{\prime}(s-2 l-2 m l+2 k l) d s
$$

we have

$$
\begin{aligned}
v(t) & =g_{2 m+1}(t)+2 \sum_{k=1}^{m}\left(g_{2 k-1}(t-2 l-2 m l+2 k l)-g_{2 k-1}((2 k-1) l)\right. \\
& =g_{2 m+1}(t)+2 \sum_{k=1}^{m} g_{2 k-1}(t-2 l-2 m l+2 k l)+2 m h .
\end{aligned}
$$

Denote by

$$
\begin{gathered}
\tilde{\xi}=\xi(t)+2 m h+2 \sum_{k=1}^{m} g_{2 k-1}(t-2 l-2 m l+2 k l) \\
D(t)=[-h, h]+\tilde{\xi}(t)
\end{gathered}
$$


where $t \in[(2 m+1) l,(2 m+2) l]$. Taking into account the inductive assumption and representation of the function $\xi(t)$, we have

$$
\tilde{\xi}(t)= \begin{cases}(1+2 m) \xi(t), & t \in\left[2 m l+l, 2 m l+\frac{5 l}{4}\right], \\ \xi(t)+4 m h, & t \in\left[2 m l+\frac{5 l}{4}, 2 m l+\frac{3 l}{2}\right], \\ \xi(t)+4 m h+2 m \xi(t), & t \in\left[2 m l+\frac{3 l}{2}, 2 m l+\frac{7 l}{4}\right], \\ \xi(t), & t \in\left[2 m l+\frac{7 l}{4}, 2 m l+2 l\right] .\end{cases}
$$

Since $v(t)$ is the solution of the problem

$$
\left\{\begin{array}{l}
-v^{\prime}(t) \in N_{D(t)}(v(t)), t \in[(2 m+1) l,(2 m+2) l] \\
v((2 m+1) l)=-h
\end{array}\right.
$$

one has

$$
v(t)= \begin{cases}-h+\xi(t)+2 m \xi(t), & t \in\left[2 m l+l, 2 m l+\frac{5 l}{4}\right], \\ h+4 m h, & t \in\left[2 m l+\frac{5 l}{4}, 2 m l+\frac{3 l}{2}\right], \\ \xi(t)+4 m h+2 m \xi(t)+h, & t \in\left[2 m l+\frac{3 l}{2}, 2 m l+\frac{7 l}{4}\right], \\ -h, & t \in\left[2 m l+\frac{7 l}{4}, 2 m l+2 l\right] .\end{cases}
$$

Hence, $g_{2 m+1}(t)=v(t)-\tilde{\xi}(t)+\xi(t)$ and we get expression (4.4) for the function $g_{2 m+1}(t)$. Other cases can be analyzed in the same way. Denote by $g(t)=g_{i}(t)$, where $t \in[i l,(i+1) l]$, $i=0,1,2, \ldots$. Since $u(l, t)=g(t)$, we have that the right end of the string periodically moves with the period 1 , starting at time $t=\frac{l}{4}$. Rewrite problem (4.1) as

$$
\left\{\begin{array}{l}
\frac{\partial^{2} u}{\partial x^{2}}=\frac{\partial^{2} u}{\partial t^{2}} \\
u(x, 0)=0 \\
\partial u \\
\frac{\partial t}{\partial t}(x, 0)=0 \\
u(0, t)=0 \\
u(l, t)=g(t)
\end{array}\right.
$$

The solution of the last problem can be represented as

$$
u(x, t)=\sum_{n=0}^{\infty} \underline{g}(t-(2 n+1) l+x)-\sum_{n=0}^{\infty} \underline{g}(t-(2 n+1) l-x),
$$

where

$$
\underline{g}(t)= \begin{cases}g(t), & t \geq 0 \\ 0, & t<0 .\end{cases}
$$

In view of equality (4.6), the sums (4.5) have only a finite number of terms. Let us show that the function $u\left(\frac{l}{2}, t\right)$ is $2 l$-periodic. We have

$$
u\left(\frac{l}{2}, t\right)=\sum_{n=0}^{\infty} \underline{g}\left(t-2 n l-\frac{l}{2}\right)-\sum_{n=0}^{\infty} \underline{g}\left(t-2 n l-\frac{3 l}{2}\right) .
$$

On the other hand,

$$
\begin{aligned}
u\left(\frac{l}{2}, t+2 l\right) & =\sum_{n=0}^{\infty} \underline{g}\left(t-2 n l+\frac{3 l}{2}\right)-\sum_{n=0}^{\infty} \underline{g}\left(t-2 n l+\frac{l}{2}\right) \\
& =\underline{g}\left(t+\frac{3 l}{2}\right)-\underline{g}\left(t+\frac{l}{2}\right)+u\left(\frac{l}{2}, t\right) .
\end{aligned}
$$


Since

$$
\underline{g}\left(t+\frac{3 l}{2}\right)=g\left(t+\frac{3 l}{2}\right)=g\left(t+\frac{l}{2}\right)=\underline{g}\left(t+\frac{l}{2}\right),
$$

we obtain $u\left(\frac{l}{2}, t\right)=u\left(\frac{l}{2}, t+2 l\right)$. Let us show that $u\left(\frac{l}{4}, t\right)=k h$ for $t=k l$, where $k \in N$. We have

$$
u\left(\frac{l}{4}, t\right)=\sum_{n=0}^{\infty} \underline{g}\left(t-2 n l-\frac{3 l}{4}\right)-\sum_{n=0}^{\infty} \underline{g}\left(t-2 n l-\frac{5 l}{4}\right) .
$$

Let $k$ be an even number. It follows that

$$
\begin{aligned}
u\left(\frac{l}{4}, k l\right)= & \sum_{n=0}^{\frac{k}{2}-1} \underline{g}\left(k l-2 n l-\frac{3 l}{4}\right)-\sum_{n=0}^{\frac{k}{2}-1} \underline{g}\left(k l-2 n l-\frac{5 l}{4}\right) \\
= & \left(\underline{g}\left((k-1) l+\frac{l}{4}\right)+\underline{g}\left((k-3) l+\frac{l}{4}\right)+\ldots+\underline{g}\left(l+\frac{l}{4}\right)\right) \\
& -\left(\underline{g}\left((k-2) l+\frac{3 l}{4}\right)+\underline{g}\left((k-4) l+\frac{3 l}{4}\right)+\ldots+\underline{g}\left(\frac{3 l}{4}\right)\right) \\
= & \frac{k}{2}\left(\underline{g}\left(\frac{l}{4}\right)-\underline{g}\left(\frac{3 l}{4}\right)\right)=k h .
\end{aligned}
$$

Let $k$ be an odd number. Then

$$
\begin{aligned}
u\left(\frac{l}{4}, k l\right)= & \sum_{n=0}^{\frac{k-1}{2}} \underline{g}\left(k l-2 n l-\frac{3 l}{4}\right)-\sum_{n=0}^{\frac{k-1}{2}-1} \underline{g}\left(k l-2 n l-\frac{5 l}{4}\right) \\
= & \left(\underline{g}\left((k-1) l+\frac{l}{4}\right)+\underline{g}\left((k-3) l+\frac{l}{4}\right)+\ldots+\underline{g}\left(\frac{l}{4}\right)\right) \\
& -\left(\underline{g}\left((k-2) l+\frac{3 l}{4}\right)+\underline{g}\left((k-4) l+\frac{3 l}{4}\right)+\ldots+\underline{g}\left(\frac{3 l}{4}+l\right)\right. \\
= & \frac{k}{2}\left(\underline{g}\left(\frac{l}{4}\right)-\underline{g}\left(\frac{3 l}{4}\right)\right)+\frac{1}{2} \underline{g}\left(\frac{l}{4}\right)+\frac{1}{2} \underline{g}\left(\frac{3 l}{4}\right)=k h .
\end{aligned}
$$

Thus the function $u\left(\frac{l}{4}, t\right)$ is not periodic in $t$. Similarly, we obtain if $x=\frac{3 l}{4}$, then $u\left(\frac{3 l}{4}, k l\right)=-k h$.

\section{Acknowledgments}

The first and the third authors were supported by the Ministry of Education and Science of the Russian Federation in the frameworks of the project part of the state work quota [project number 1.3464.2017], the joint Taiwan MOST-Russia RFBR grant No.17-51-52022.

\section{REFERENCES}

[1] L. Adam, J. Outrata, On optimal control of a sweeping process coupled with an ordinary differential equation, Discrete Contin. Dyn. Syst. Ser. B 19 (2014), 2709-2738.

[2] S. Adly, B. K. Le, Unbounded second-order state-dependent Moreau's sweeping processes in Hilbert spaces, J. Optim. Theory Appl. 169 (2016), 407-423.

[3] C. Castaing, M. Monteiro Marques, BV periodic solutions of an evolution problem associated with continuous moving convex sets, Set-Valued Anal. 3 (1995), 381-399.

[4] M.V. de Hoop, P. Kepley, L. Oksanen, An exact redatuming procedure for the inverse boundary value problem for the wave equation, SIAM J. Appl. Math. 78 (2018), 171-192.

[5] J.F. Edmond, L. Thibault, Relaxation of an optimal control problem involving a perturbed sweeping process, Math. Program. 104 (2005), 347-373. 
[6] A. Fiscella, E. Vitillaro, Blow-up for the wave equation with nonlinear source and boundary damping terms, Proc. R. Soc. Edinb. Sect. A. 145 (2015), 759-778.

[7] G.R. Goldstein, J.A. Goldstein, G. Reyes, Overdamping and energy decay for abstract wave equations with strong damping, Asymptotical Anal. 88 (2014), 217-232.

[8] V.A. Il'in, Two-end point boundary control of ibrations described by a finite energy generalized solution of the wave equation, Differential Equations 36 (2000), 1659-1675.

[9] V.A. Il'in, Boundary control of oscillations at one endpoint with the other endpoint fixed in terms of a finiteenergy generalized solution of the wave equation, Differential Equations 36 (2000), 1832-1849.

[10] V. A. Il'in, E. I. Moiseev, Optimization of boundary controls of string vibrations, Russian Math. Surveys 60 (2005), 1093-1119.

[11] M. Kamenskii, O. Makarenkov, On the pesponse of autonomous sweeping processes to periodic perturbations, Set-Valued Var. Anal. 24 (2016), 551-563.

[12] M. Kamenskii, O. Makarenkov, L. N. Wadippuli, P. Raynaud de Fitte, Global stability of almost periodic solutions to monotone sweeping processes and their response to non-monotone perturbations, Nonlinear Anal. 30 (2018), 213-224.

[13] M. Kamenskii, C.F. Wen, M. Zvereva, A string oscillations simulation with boundary conditions of hysteresis type, Optimization 67 (2018), 1321-1332.

[14] M. Kamenskii, Y.C. Liou, C.F. Wen, M. Zvereva, On a hyperbolic equation on a geometric graph with hysteresis type boundary conditions, Optimization (2019), doi: 10.1080/02331934.2018.1561694.

[15] M. Kamenskii, C.F. Wen, Z. Zalukaeva, M. Zvereva, The influence function properties for a problem with discontinuous solutions, Appl. Anal. Optim. 1 (2017), 259-281.

[16] P. Krejci, T. Roche, Lipschitz continuous data dependence of sweeping processes in BV spaces, Discrete Contin. Dyn. Syst. B 15 (2011), 637-650.

[17] M. Kunze, M. Monteiro Marques, An introduction to Moreau's sweeping process, Impacts in Mechanical Systems Lecture Notes in Physics 551 (2000), 1-60.

[18] A.N. Tikhonov, A.A. Samarskii, Equations of Mathematical Physics, Dover Publication, New York, 1990.

[19] E. Vitillaro, On the wave equation with hyperbolic dynamical boundary conditions, interior and boundary damping and source, Arch. Ration. Mech. Anal. 223 (2017), 1183-1237.

[20] M. Zvereva, A string oscillations simulation with nonlinear conditions, Mem. Differ. Equ. Math. Phys. 72 (2017), 141-150. 